\title{
OPTIMIZATION OF INTELLIGENT ILLUMINATION IN UNIVERSITY CLASSROOM BASED ON FMRAS CONTROL ALGORITHM
}

\author{
Wenhao DUN \\ School of Civil Engineering \& Architecture of Wuhan University of Technology / \\ Hubei Institute of Fine Arts, Wuhan, Hubei, China; \\ E-mail: ebyltr@163.com
}

\begin{abstract}
Based on the FMRAS control algorithm, the optimization of the intelligent illumination in the classroom was studied, aiming at optimizing the classroom illumination and improving the energy utilization. The FMRAS control algorithm, based on technology, was used to design a fuzzy control system of intelligent illumination in college classroom. The analysis is based on the improved adaptive algorithm - FMRAS. After the PWM code is automatically generated, the system can automatically control the lighting of the lamps. Through the research, the result has been obtained: the method designed can meet the needs of the optimization of intelligent lighting in the classroom, and can further improve the efficiency and intelligence of classroom lighting in universities.

Keywords: fuzzy model reference adaptive system (FMRAS) control algorithm, college classrooms, intelligent lighting

\section{INTRODUCTION}

China has put energy saving work as the focus of the " $11^{\text {th }}$ Five-Year" work. The call for "building a conservation oriented society" was put forward. The Ministry of education has also actively responded to the call for "building a conservation oriented campus". As an important place for college teaching, the university classroom usually adopts the open management model. Students basically
\end{abstract}

have no fixed classrooms and seats, while the building managers are only responsible for health or security [1]. Therefore, there is a waste of lights, unturned lights and less people because of the large number of lamps and lanterns in the classroom and the long time of use. The proportion of lighting in the whole school's electricity consumption has always been high. If a school has 200 classrooms in accordance with the $100 \mathrm{~m}^{2}$ [2], the intelligent lighting control is adopted to make the electricity saving rate of $30 \%$, and the electricity fee is saving from 150 to 200 thousand yuan per year. The above estimates are not including in the line loss and the ageing of the luminaire [3]. The above data show that the energy saving work in the classroom is a long-term and arduous task. The intelligent lighting control in university classrooms should not only meet the requirements of indoor lighting, but also prolong the life of lamps and lanterns, reduce operation costs, simplify distribution control devices and reduce wiring workload under the premise of energy saving [4]. A new wireless network technology with short distance, low speed, low complexity, low power consumption and low cost Zigbee technology has its unique advantages: power saving, reliability, short delay, large network capacity, high security, licensed frequency band, self-organization and so on [5]. It is very suitable for the wireless connection of the sensor nodes in the classroom. It not only conforms to the characteristics of the school network communication, but also saves a lot of manpower and material resources. There- 
fore, the research and Realization of intelligent lighting control system has very important practical significance.

\section{STATE OF THE ART}

The key technology of intelligent lighting system is information transmission and intelligent control. The power carrier technology, the integrated wiring technology and the wireless network technology are the three main technologies of the information transmission in the current intelligent lighting control system in China. The integrated wiring technology connects the central controller, the switch, and the terminal with the unshielded twisted pair and the optical cable as the medium [6]. The central controller transfers the wireless signals sent by the remote control to the terminals in a wired way, and the terminal completes the corresponding instructions. This method needs to set up the extra weak power control line to achieve the stable signal, which is more suitable for the large area control of the building and the district intelligence. But there are the following shortcomings: the specialized technical installation and maintenance large amount of engineering, long installation cycle, high cost, and poor compatibility of equipment. The wireless network realizes intercommunication and intelligent control of all kinds of electronic devices through infrared, Bluetooth, Zigbee and other technologies [7]. Wireless networks can provide greater mobility and convenience, which makes the use of space more flexible. The cost and energy spent on the generic cabling were saved. The application of wireless network technology to the school network has become an unstoppable trend. As a technology of low power consumption, low data rate and low cost, Zigbee is more suitable for classroom lighting automation system and low-cost equipment for low rate rate transmission. Zigbee is the best choice for intelligent lighting control system in Colleges and Universities [8].

\section{METHODOLOGY}

\subsection{Design of Illumination Acquisition Module and Vibration Sensing Module}

First of all, it is designed for the optical illumination acquisition module, which fully uses natural light. The combination of natural light and light is not only an effective way to save energy, but also to create a good visual effect. Therefore, a light illumination acquisition module is designed, and its structure diagram is shown in Fig. 1.

The module consists of three parts, which are data acquisition, data processing and PWM drive control. Among them, data processing and driving control are mainly completed by CC2430. The illuminance sensor is used by ON Company to produce a visible light sensor (ON9658). The sensor is an optoelectronic integrated sensor, with a built-in UNIKA sensor receiver, which automatically attenuates near infrared. The spectral response is close to the human eye function. It is highly sensitive in the visible range, and the output current varies linearly with illumination. The main function is to collect the bad illuminance signal of the classroom and send the signal to the data processing unit through the digital I/O pin P0.7 of the CC2430, whose circuit is realized as shown in Fig. 2.

As the core of data processing and driving control, Zigbee chip CC2430-F128 integrates ADC with 14 bit modulus / number conversion, and it can write the result of conversion to memory controller through DMA mode without CPU interference. This cannot only make the detection results more accurate, but also improve the overall efficiency of the system and reduce the power consumption. Configure the 16 bit timer of the CC2430, TIMER1.



Fig.1. Structure diagram of illuminance acquisition module 


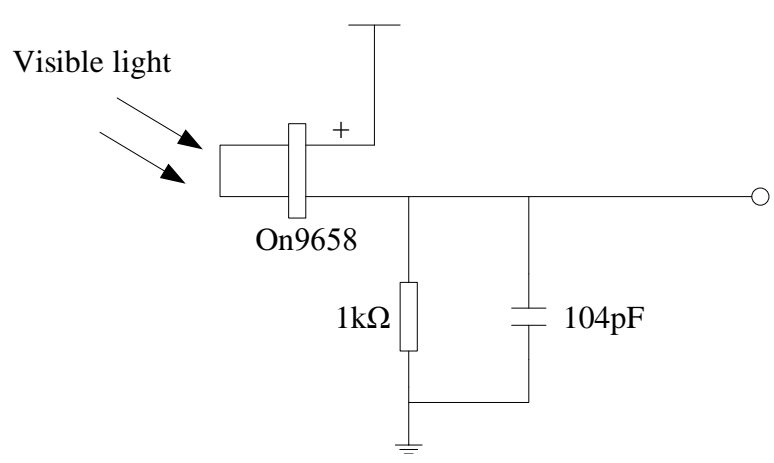

Fig.2. Optical illumination acquisition module circuit

The centre aligned PWM output can be generated. First, TIMER1 is selected as a positive count / down count mode, and the channel output comparison mode selects 5. Secondly, the period of the PWM signal is stored in the T1CC0. Finally, the PWM duty ratio is calculated according to the control changes of the fuzzy inference output. It is used to adjust the effective value of the output voltage to compensate the natural light illumination to stabilize the light environment of the classroom.

The second is the design of the vibration sensing module. At present, the multimedia classrooms in colleges and universities are quite common, and such classrooms have special requirements for the illumination. It is not only to meet the students' reading and note taking, but also to ensure the clarity of the projection screen. So, the vibration sensing module was designed and installed in the projector. When the fan is rotated, the module senses and determines that the classroom is in the projective state. At the same time, the lamp in the rostrum area is closed, while the reserved seat area keeps $50 \%$ of the standard illuminance. The circuit diagram of this module is shown in Fig. 3.

In this design, the vibration sensing element, SW-180-10P, is used as a type of non-directional vibration induction device, which can be triggered at any angle. It is the open OFF state at any angle at rest. When the external force is collided or vibrate, the spring deformation and the contact of the central electrode make the two pins turn into the ON state instantaneously. When the external force is disap-



Fig.3. Vibration sensor module

pearing, the circuit is restored to the open OFF state. Considering the cost factor, the LMV324 operational amplifier is used as the comparator in this design. Its integration makes it smaller than the independent comparator occupying the circuit board, thus further saving the cost.

\subsection{Adaptive FMRAS Algorithm Based on Fuzzy Control System Design}

Fuzzy control is a kind of computer model based on fuzzy set theory; fuzzy language is variable and fuzzy logic reasoning. Fuzzy control system is a digital automatic control system based on fuzzy mathematics, fuzzy language form knowledge representation and fuzzy logic rule inference, and a closed loop structure with feedback channel, which is composed of computer control technology. The structural form of its system is shown in Fig. 4.

The fuzzy control system is usually composed of five parts, such as fuzzy controller, input / output interface, actuator, wave control object and detection device (sensor).

The basic structure of the fuzzy controller is shown in Fig. 5.

As shown in Fig. 5, in this system, fuzzy adaptive mechanism produces a fuzzy adaptive signal based on the difference between the output of YM and the output YF of the controlled system according to the reference model, controlling the output of the controlled system to the reference model output. However, in the actual situation, there will be changes in the object parameters, state interference

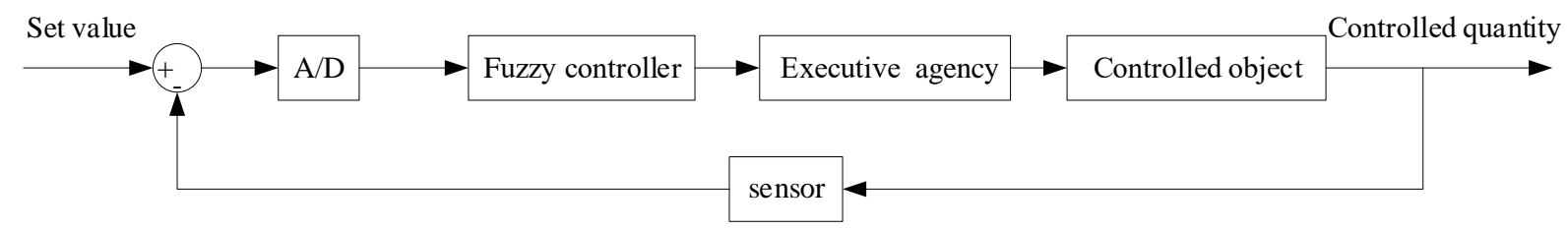

Fig.4. Structure block diagram of fuzzy control system 


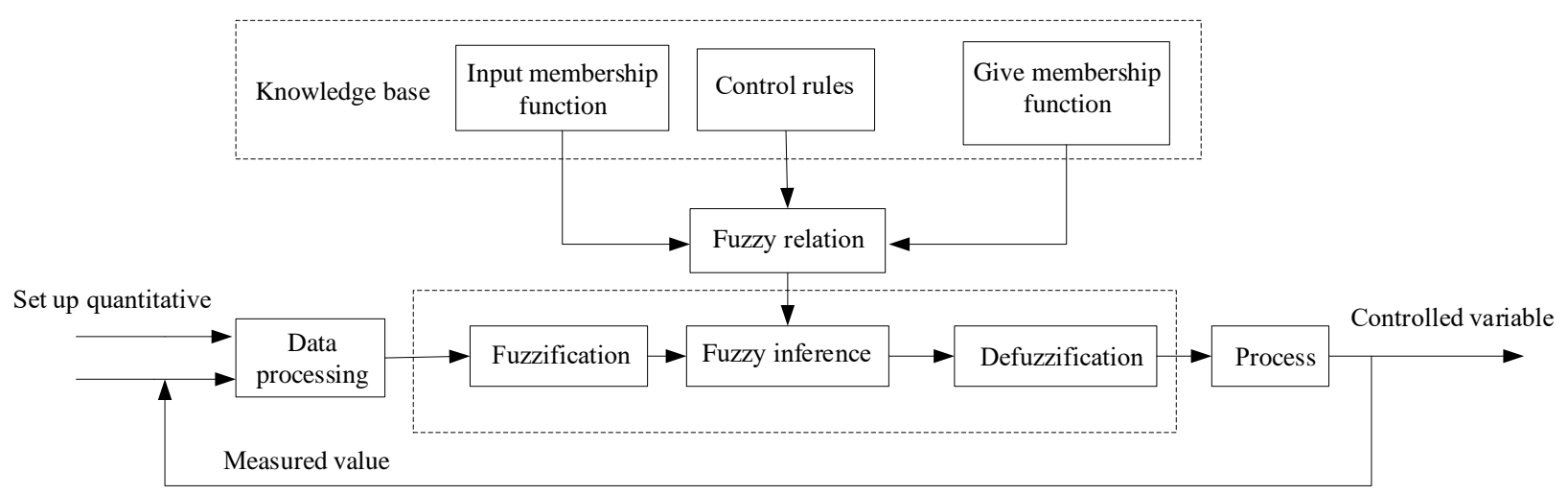

Fig.5. Fuzzy control structure diagram

and so on. Therefore, the T-S fuzzy model is adopted in this mechanism, and the following implication is adopted.

$$
\text { If } e=A \text { and } e c=B \text { then } u=g(e) \text {. }
$$

Among them, $A$ and $B$ are fuzzy subset; $g(e)$ is a continuous function of $e$, as shown in Table 1 .

g1(e), g2(e), g3(e) in the table are K0e, $0.6 \mathrm{KOe}$ and $0.3 \mathrm{KOe}$, respectively. $\mathrm{KO}$ is the ratio coefficient. According to the fuzzy inference algorithm, the adaptive fuzzy query can be obtained as shown in Table 2.

The product of any element in the table and the K0e is the output Uf of the fuzzy adaptive mechanism of the corresponding system state. Obviously there is:

$$
U f=f(e, e c) K 0 e .
$$

In the form, $f$ represents a kind of nonlinearity, whose value can be queried in Table 2. The Matlab's Fuzzy Logic Toolbox provides a seamless con- nection with Simulink. After the fuzzy inference system is established in the fuzzy logic toolbox, it can be simulated and analyzed in the Simulink simulation environment immediately. In Simulink, there is a corresponding block diagram of fuzzy logic control, and the block diagram is copied to the Simulink simulation model established by the user. The name of the fuzzy reasoning matrix of the block diagram of the fuzzy logic controller is the same as the name of the fuzzy inference system which is established by the user in the Matlab workspace. The connection between the fuzzy inference system and the Simulink can be completed. The core part of the model is described as follows: the step signal issued by Step represents the set value of the standard illuminance. In this simulation, it is set as $400 \mathrm{~lx}$. Considering the diversity of the light source, the controlled model uses the first order and the two order two transfer functions to verify the practicability of the algorithm. The reference model describes the dynamics of the controlled object or represents an ideal dynamic, and does not need to establish an accurate mathematical model. In this design, the

Table 1. Fuzzy Rules Table of Fuzzy Adaptive Mechanism

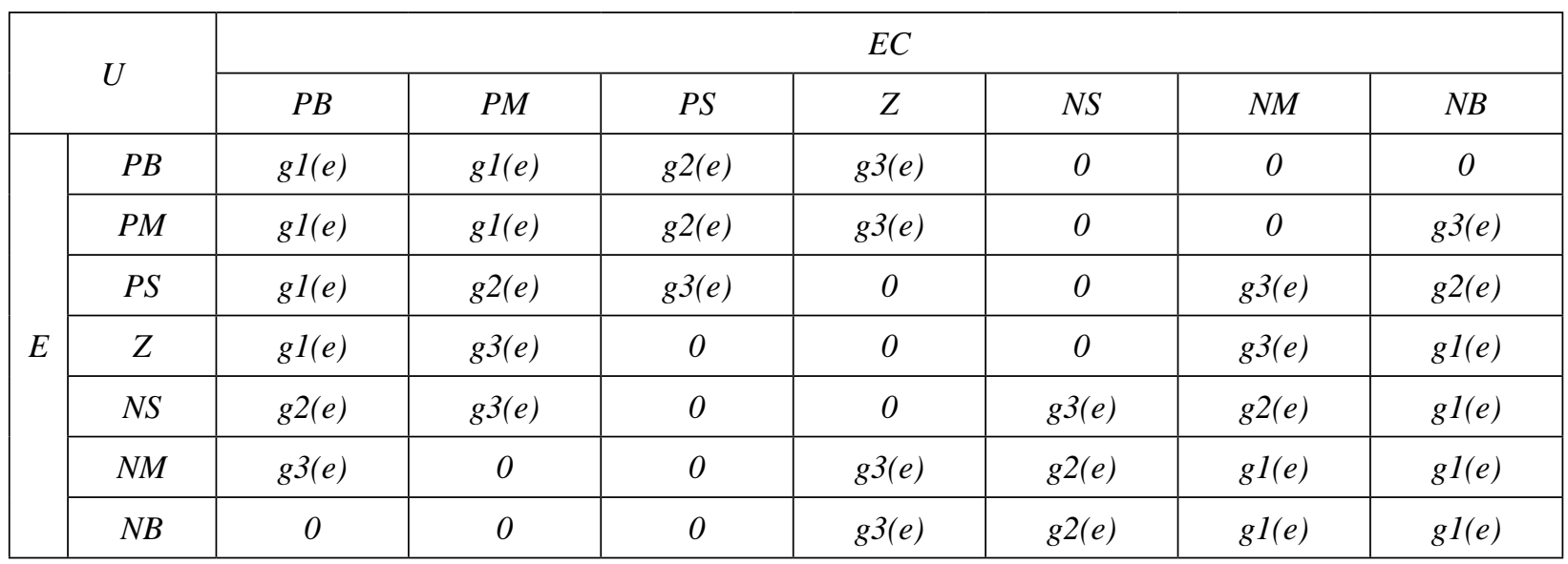


Table 2. Fuzzy Self-Adaptive Mechanism Fuzzy Query Table

\begin{tabular}{|c|c|c|c|c|c|c|c|c|c|c|c|c|c|c|}
\hline & \multirow{2}{*}{$f$} & \multicolumn{13}{|c|}{ ec } \\
\hline & & -6 & -5 & -4 & -3 & -2 & -1 & 0 & 1 & 2 & 3 & 4 & 5 & 6 \\
\hline \multirow{13}{*}{$e$} & -6 & 1 & 1 & 0.93 & 0.82 & 0.66 & 0.46 & 0.38 & 0 & 0 & 0.3 & 0.3 & 0.3 & 0.3 \\
\hline & -5 & 1 & 1 & 0.9 & 0.81 & 0.65 & 0.45 & 0.37 & 0 & 0 & 0.3 & 0.3 & 0.3 & 0.3 \\
\hline & -4 & 0.97 & 0.95 & 0.87 & 0.77 & 0.62 & 0.44 & 0.36 & 0 & 0.1 & 0.32 & 0.35 & 0.38 & 0.4 \\
\hline & -3 & 0.96 & 0.91 & 0.85 & 0.67 & 0.53 & 0.41 & 0.35 & 0 & 0.1 & 0.34 & 0.38 & 0.4 & 0.43 \\
\hline & -2 & 0.92 & 0.83 & 0.71 & 0.58 & 0.5 & 0.36 & 0.34 & 0 & 0.3 & 0.36 & 0.41 & 0.46 & 0.53 \\
\hline & -1 & 0.86 & 0.77 & 0.66 & 0.48 & 0.35 & 0.3 & 0.3 & 0 & 0.3 & 0.38 & 0.47 & 0.51 & 0.6 \\
\hline & 0 & 0.8 & 0.67 & 0.5 & 0.4 & 0.3 & 0 & 0 & 0 & 0.3 & 0.4 & 0.5 & 0.67 & 0.8 \\
\hline & 1 & 0.6 & 0.51 & 0.47 & 0.38 & 0.3 & 0 & 0.3 & 0.3 & 0.35 & 0.48 & 0.66 & 0.77 & 0.86 \\
\hline & 2 & 0.53 & 0.46 & 0.41 & 0.36 & 0.3 & 0 & 0.34 & 0.36 & 0.5 & 0.58 & 0.71 & 0.83 & 0.92 \\
\hline & 3 & 0.43 & 0.4 & 0.38 & 0.34 & 0.1 & 0 & 0.35 & 0.41 & 0.53 & 0.67 & 0.85 & 0.91 & 0.96 \\
\hline & 4 & 0.4 & 0.38 & 0.35 & 0.32 & 0.1 & 0 & 0.36 & 0.44 & 0.62 & 0.77 & 0.87 & 0.95 & 0.97 \\
\hline & 5 & 0.3 & 0.3 & 0.3 & 0.3 & 0 & 0 & 0.37 & 0.45 & 0.65 & 0.81 & 0.9 & 1 & 1 \\
\hline & 6 & 0.3 & 0.3 & 0.3 & 0.3 & 0 & 0 & 0.38 & 0.46 & 0.66 & 0.82 & 0.93 & 1 & 1 \\
\hline
\end{tabular}

adaptive mechanism uses the fuzzy inference method of the post - part as the function form. This method cannot be compiled directly with a Matlab fuzzy editor. The value of Table 2 needs to be filled in the two-dimensional table Look-Up Table (2-D). At the same time, the Matlab Function dialog box is changed to four to five into the round function. The feedback fuzzy controller uses the incremental signal as the output, and then outputs the output to the controlled object. This is equivalent to the introduction of integral action, which is beneficial to eliminate static deviation.

\section{RESULT ANALYSIS AND DISCUSSION}

\subsection{Research on Fuzzy Feedback Controller}

The input / output variable of the feedback controller is determined. In this design, the feedback controller is a two dimensional fuzzy controller, which is the dual input output mode. The input variable is the illumination deviation $e$ and the deviation rate $e_{c}$. This method can not only guarantee the stability of the control system, but also reduce the overshoot and concussion.

For the determination of membership function of input / output $M$, the function of $L i$ is a fuzzy set, which should be applied to practical problems.
In this system, the common triangle membership function is used, and the Matlab fuzzy toolbox graphic interface is used to determine the function editor. For example, Fig. 6.

The domain of the input variable is used for this fuzzy controller for the selection of the domain and factor of the input / output variable. According to the experience of the expert, if the value of the system deviation $e$ is greater than $201 \mathrm{x}$, it should be "PB" for the maximum value of the language. The value of $e$ is less than equal to $-20 \mathrm{~lx}$, which is "NB". Therefore, the domain of the deviation $e$ is $[-20,20]$, and the fuzzy set domain is $[-5$, 5]. The value of the quantizing factor $K e$ is 0.25 . It is worth noting that the domain of the deviation $e$ of the input variable of the fuzzy controller is not equal to the range of the deviation in the system operation. In actual control, a limiter should be added to ensure that the error value is within the allowable range.

In the same way, the domain and its factors of the controller output and error change can be determined. According to the illuminance standard of China's civil building lighting design standard and international vision ergonomics indoor lighting system, $4201 \mathrm{x}$ is used as the standard illumination value, and the illumination area of luminaire is set to $[300,500]$. Therefore, the ratio factor $\mathrm{Ku}$ of the 




(a) The membership function of illuminance deviation $\mathrm{E}$



(b) The membership function of the variation rate of illuminance EC

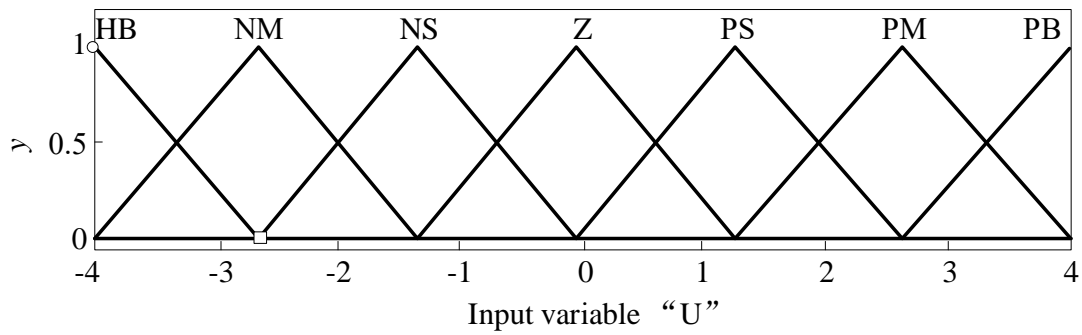

(c) The membership function of the incremental $U$ of the controlled system

Fig.6. The subordinate function of the fuzzy controller

controller output should be 5 . And the error changes need to be set according to the actual needs, $K e c=0.01$ in this controller.

In actual control, the continuous domain needs to be discretized. For example, the fuzzy set theory domain of the error $e$ is divided into 7 grades. Each level corresponds to a fuzzy subset. That is, $\{-5,-4,-3,-2,-1,0,1,2,3,4,5\}$ respectively (NB, NM, NS, ZO, PS, PM,). Then determine the membership degree of the element in the domain on the fuzzy language variable.

The fuzzy control rule is the core of fuzzy controller for the determination of the fuzzy control rule table and the descendant table, reflecting the knowledge collection of a particular control problem, such as offset. When making fuzzy rules, the following requirements should be met: intuitively, fuzzy control models should always be able to get a proper control for every process state reasoning. This feature is called "completeness". It means you can't get out of control. For the number of fuzzy control rules, if the input of fuzzy controller is $m$, the fuzzy classification number of each input is $n 1$, $n 2, \ldots, n m$, respectively. The maximum possible fuzzy rule number is $N_{\max }=n 1 n 2 \ldots n m$. How much the actual fuzzy control number should be determined depends on many factors. The general principle is that, under the condition of completeness, the number of rules is taken as much as possible so as to simplify the design and implementation of the fuzzy controller. For the consistency of fuzzy control rules, fuzzy control rules are mainly based on the experience of the operator, depending on the requirements for a variety of performance, and the requirements of different performance indicators are often mutually restrictive and even contradictory to each other. This requires the inconsistency of fuzzy control.

In the light control, the illuminance value of the light is mainly influenced by the external natural light. The natural light is high and the lamps in the classroom should be closed. Low natural illumination, room lamps and lanterns should be opened, and the standard value of illuminance is guaranteed. This is the work of the actual lighting control. According to the actual situation and the experience of artificial lighting control, the fuzzy control rule table can be obtained. In the results, $E, E C$ and $U$ are fuzzy language variables of illumination deviation $e$, deviation rate $\mathrm{EC}$ and output cancellation $u$. 


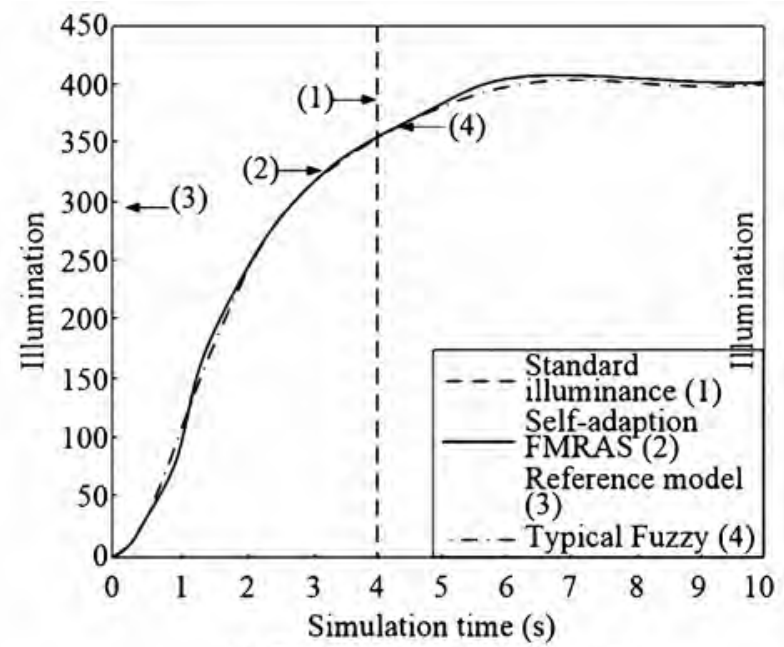

(a) First order contrast diagram

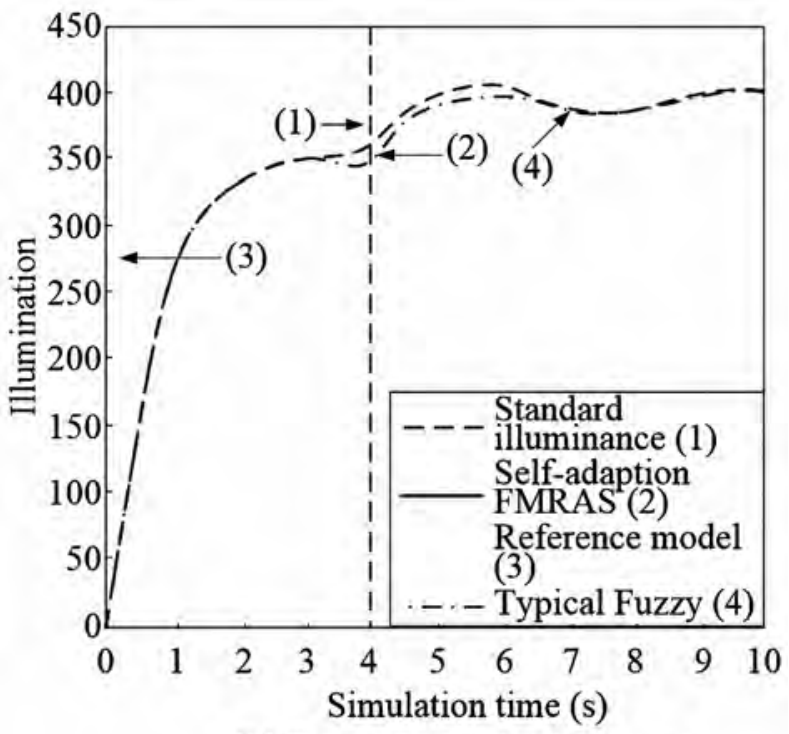

(b) Two order contrast diagram

Fig.7. Improved adaptive simulation results

This table represents the control rules: if ( $e$ is $N B$ ) and (ec is $N B$ ) then ( $U$ is $P B$ ).

In practical use, the results are converted into a fuzzy control response table. By using fuzzy logic control method, all input languages are changed into $M(e, e c)$, and the tables generated by the fuzzy controller of each state are calculated by quantized offline combinations, putting it in the form of "file" into the computer. When real-time control is carried out, the control strategy is querying from the "file" according to the input information. The fuzzy reasoning method is as follows: first, the fuzzy relation $R$ is got, and then the synthetic inference method is used to get the fuzzy output, according to the input deviation and the rate of deviation change. Finally, the fuzzy quantity is transformed into the exact quantity after the anti fuzzification. In this case, the design of the fuzzy feedback controller is basically completed.

\subsection{Results and Analysis of Simulation}

After setting the simulation step and so on, the simulation can be carried out. To verify the progressiveness and experimentation of the improved model, the simulation is compared with the classic fuzzy control and the improved adaptive FMRAS. And the control rules of the input / output variables are guaranteed, and the quantitative factors are the same. The simulation results are shown in Fig.7. Among them, Fig. 7 (a) is the result of the performance comparison between the improved adaptive FMRAS and the classical fuzzy control under the first order system, (b) is a simulation result of the performance comparison between the improved adaptive FMRAS and the classical fuzzy control under the two order system.

From the results, we can see that adaptive FMRAS has better tracking performance, higher steady-state accuracy, smaller overshoot and higher response speed than the typical fuzzy controllers currently used, which can guarantee the requirement of classroom illumination and achieve the design goal.

\section{CONCLUSION}

The intelligent lighting control system of college classrooms is an important way to save energy in colleges and universities. Zigbee technology, embedded ARM technology, sensor technology and other lighting systems were combined. The intelligent lighting control system of college classrooms based on Zigbee was designed, relying on illuminance sensor, vibration sensor and human body infrared sensor to automatically collect information such as illuminance, usage state, number and location of personnel. And the modified adaptive FMRAS (Fuzzy Model Reference Learning Control) control model was used for fusion analysis, and the PWM code was automatically generated for intelligent controls lamps and lanterns. On the premise of meeting the national standard of illuminance, the purpose of saving energy was achieved. Considering the convenience of management and friendly man-machine interaction principle, LCD display and monitoring management system were 
attached to facilitate monitoring and viewing of managers, teachers and students. The function of making lamps and lanterns achieve standard illumination automatically was realized, quickly and stably based on indoor personnel location, natural illumination and classroom usage state. At the same time, the service life of the lamps and lanterns was prolonged, the workload of wiring was reduced, and the purpose of energy saving was achieved.

\section{REFERENCES:}

1. Zhang Y., Li B., Su X., et al. A Study of Schoolroom Lighting Fuzzy Control System. International Journal of Control and Automation, 2015. V8, \#1, pp.189-196.

2. Morey M S., Virulkar V B., Dhomane G A. MRAS based Speed identification and online updating of rotor time constant for sensorless field oriented controlled induction motor. Emerging Trends in Electrical Electronics \& Sustainable Energy Systems (ICETEESES), International Conference on. IEEE, 2016. pp.179-185.
3. Kirkby, J. L., Deng, S. "Static hedging and pricing of exotic options with payoff frames." Available at SSRN2501812, 2017.

4. Zumbrunn S., McKim C., Buhs E., et al. Support, belonging, motivation, and engagement in the college classroom: A mixed method study. Instructional Science, 2014. V42, \#5, pp.661-684.

5. Biddix J P., Chung C J., Park H W. The hybrid shift: Evidencing a student-driven restructuring of the college classroom. Computers \& Education, 2015. V80, pp.162-175.

6. Berry M J., Westfall A. Dial D for distraction: The making and breaking of cell phone policies in the college classroom. College Teaching, 2015. V63, \#2, pp.62-71.

7. Georgieva D., Schledermann K M., Nielsen S M L., et al. Designing User Centred Intelligent Classroom Lighting. Interactivity, Game Creation, Design, Learning, and Innovation. Springer, Cham, 2017. pp.314-323.

8. Wei W., Tao Y., Baosen T., et al. Design of intelligent multifunctional LED lighting system. Microcomputer \& Its Applications, 2016. V15, p. 31.

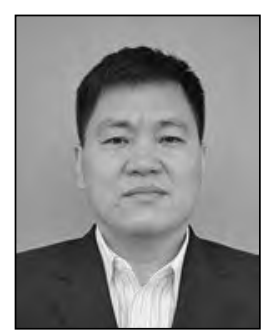

\section{Wenhao DUN,}

On-the-job Doctorate, Lecturer. Graduated from the School of Civil Engineering and Architecture of Wuhan University of Technology. The research direction is lighting design for underground engineering. He is the Fellow of Indian Society of Lighting Engineers (ISLE), member of The Institution of Engineers (India), and member of IESNA 